\title{
Cleavage of the Nuclear Matrix Protein NuMA during Apoptosis
}

\author{
Catherine Gueth-Hallonet, Klaus Weber, and Mary Osborn ${ }^{1}$ \\ Department of Biochemistry, Max Planck Institute for Biophysical Chemistry, D-37070 Goettingen, Germany
}

\begin{abstract}
NuMA is a component of the nuclear matrix which may play a structural role in the architecture of the interphase nucleus. During apoptosis NuMA is redistributed within the nucleus and is proteolysed from a 238-kDa form to a 180- to 200-kDa form. Here we show that the cleavage site leading to the stable fragment occurs between residues 1701 and 1725 . Both the changes in morphology associated with apoptosis and the cleavage of NuMA were retarded by treatment with TPCK but not by treatment by other protease inhibitors including ICE inhibitor II. ๑ 1997 Academic Press
\end{abstract}

\section{INTRODUCTION}

Apoptosis or programmed cell death is an active intrinsic process through which cells kill themselves [1]. It is a fundamental feature of animal cells essential for regulating cell populations [2]. The main distinctive morphological features of apoptosis are cell rounding and shrinking, condensation and fragmentation of the nucleus, cytoplasmic blebbing, and disassembly of the dead cell through budding of apoptotic bodies [3, 4].

The downstream effectors of apoptosis in nuclei may be ubiquitous since apoptotic cell-free extracts induce apoptosis in nuclei from a wide range of cultured cells. Nonnucleolar nuclear matrix-associated proteins, including a 126-kDa polypeptide [5] and the 13-kDa component of the $U_{1}$ SnRNP particle [6], relocate within the nucleus during apoptosis. Nuclear matrix proteins topoisomerase I and II and lamins [7, 8] are cleaved during apoptosis. NuMA, a ubiquitous nuclear matrix protein which relocates to the spindle poles during mitosis $[9,10]$, is also proteolytically processed during apoptosis $[11,12]$ indicating that it could be a structural target for a death protease. Here we analyze the position of the cleavage site and assess whether this cleavage is affected by protease inhibitors.

\section{METHODS}

Apoptosis. Apoptosis was induced in HeLaSS6 and BHK cells grown in DME medium plus $10 \%$ fetal calf serum (i) by hydroxyurea

\footnotetext{
${ }^{1}$ To whom correspondence and reprint requests should be addressed. Fax: +49551201 1578.
}

(HU, final concentration $2.5 \mathrm{mM}$, Sigma) in medium for $5 \mathrm{~h}$, Staurosporine (St, $200 \mathrm{ng} / \mathrm{ml}$, Sigma) was then added for a further 9 to 12 h, or (ii) by tumor necrosis factor $\alpha$ (TNF $\alpha, 3200$ units/ml, Signal Transduction Laboratories) and cycloheximide (10 $\mu \mathrm{g} / \mathrm{ml}$, Sigma) for $12 \mathrm{~h}$.

Mutant NuMA cDNA constructs. Full-length $(F L)$ and the new d1 and d2 NuMA cDNAs (Fig. 1) were derived from pCMV5 NuMA expression vectors [13]. Amino acid residues 7 to 1502 were deleted by digesting the pCMV5 vector containing NuMA cDNA with Smal and religating the appropriate digest products. The FL NuMA sitedirected mutant was obtained using the ExSite PCR-based site-directed mutagenesis kit (Stratagene). Ligated and mutated regions were checked by sequencing.

Transfection. BHK cells were transiently transfected using the modified calcium phosphate precipitation technique [14]. Cells were allowed to recover from transfection in fresh medium for $6 \mathrm{~h}$ before apoptotic induction.

Immunofluorescence microscopy. Immunofluorescence was by standard procedures using monoclonal NuMA SPN-3 and SPN-7 antibodies [9] as undiluted hypridoma supernatants. To detect cell death in situ, apoptotic cells were labeled with dUTP - FITC for $1 \mathrm{~h}$ with the TUNEL - FITC kit (Boehringer Mannheim) prior to NuMA staining. DNA was stained with Hoechst 33342.

Gel electrophoresis and immunoblotting. Cells from a single confluent $60-\mathrm{mm}$-diameter petri dish were scraped into $100 \mu \mathrm{l}$ doublestrength sample buffer and boiled at $100^{\circ} \mathrm{C}$. SDS-polyacrylamide gel electrophoresis and immunoblotting on nitrocellulose were performed using standard procedures. I mmunostaining of blots was performed using undiluted hybridoma supernatant for the SPN-3 and SPN-7 antibodies and peroxidase-labeled anti-mouse antibodies (Dako, Hamburg) diluted 1:5000 in the ECL technique (Amersham).

Use of protease inhibitors. HeLa cells were treated with $2.5 \mathrm{mM}$ $\mathrm{HU}$ for $9 \mathrm{~h}$. Then staurosporine and protease inhibitors were added. Final concentrations were $0.1 \mathrm{mM}$ for TLCK and TPCK (Sigma), 20 $\mu \mathrm{M}$ for ICE inhibitor II (AC-YVAD-cmk, Bachem), and $10 \mu \mathrm{M}$ for $\mathrm{E}$ 64 (Sigma).

\section{RESULTS}

\section{Induction of Apoptosis}

We induced apoptosis in HeLa cells using a 5-h incubation in hydroxyurea containing medium followed by a 9-h treatment with the protein-kinase inhibitor staurosporine. The $\mathrm{HU}$ treatment blocked the cells in interphase (Fig. 2A), while the subsequent staurosporine treatment induced a drastic change in morphology, giving rise to small round cells which protruded from the substratum but did not detach (Fig. 2B [15]). $\mathrm{H}$ oechst staining of such cells showed patches of highly condensed chromatin located predominantly at the nuclear margin (Figs. 2E and 2G). These changes in cell 


\section{FL NuMA (238 kDa)}

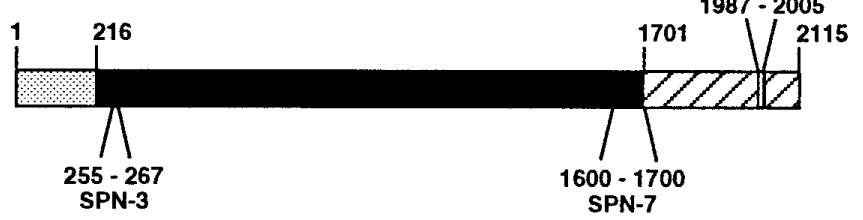

d1 NuMA (26 kDa)

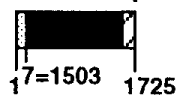

d2 NuMA (23 kDa)

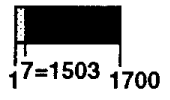

FIG. 1. Schematic representation of expressed recombinant wild-

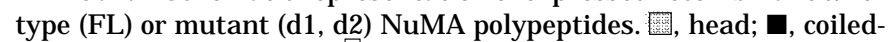
coil rod domain; $\mathbb{Z}$,tail; $]$, nuclear localization signal (NLS). EXpected molecular weights are given in parentheses. The SPN-3 and SPN-7 epitopes are given under the FL sequence. The amino acid numbering system is from Yang and Snyder [18].

shape and chromatin state and distribution correspond to changes normally seen during apoptosis [3, 4]. The apoptotic state was further demonstrated using an in situ TUNEL (terminal deoxynucleotidyltransferasemediated dUTP nick end labeling) assay. TUNEL detected all cells showing condensation of the chromatin but did not stain cells showing a normal Hoechst staining (Figs. $2 \mathrm{G}$ and $2 \mathrm{H}$ ). The long stability of the treated cells (more than $24 \mathrm{~h}$ ), the absence of cell blebbing, the persistence of the nuclear lamina, and the absence of a typical DNA ladder reflecting internucleosomal fragmentation (although such a ladder is detected in HL60 cells under the same conditions) suggested an incomplete apoptotic state, which could not be reversed by growth in normal medium without drugs.

\section{NuMA Location Changes during Apoptosis}

In untreated cells, NuMA is found throughout the nucleoplasm, with the exception of the nucleolus, in a distribution which approximates that of chromatin (F igs. 2C and 2D). In apoptotic cells, NuMA was clearly excluded from both the condensed chromatin masses and from the nucleoli (Figs. 2E and $2 \mathrm{~F}$ ).

\section{Proteolysis of NuMA during Apoptosis}

To see whether the change in location of NuMA during apoptosis could be linked to specific proteolytic cleavage, we immunoblotted the whole cell extracts (Fig. 3A). In control Hela cells, SPN-3 antibody revealed a 240-kDa band corresponding to the FL NuMA protein (lane 1 ). In cells treated with $\mathrm{HU}+$ St, SPN-3 antibody detected both the FL protein and a smaller 190kDa fragment (lane 2). The 190-kDa form was not
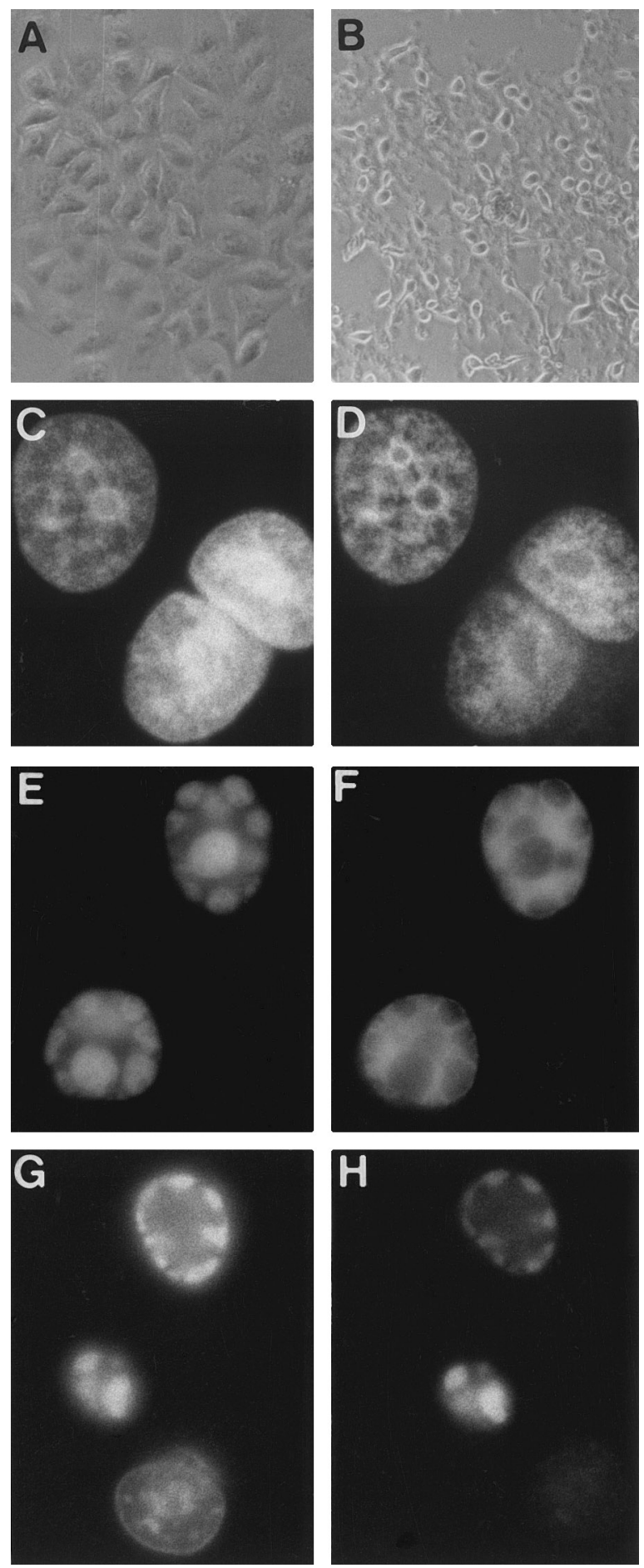

FIG. 2. (A, B) Phase-contrast microscopy of live HeLa cells. Note the absence of round mitotic cells after 5-h treatment with $2.5 \mathrm{~mm}$ hydroxyurea (A). After treatment with staurosporine for an additional $9 \mathrm{~h}$ many cells are rounded (B). (C-F) Hoechst stain (C, E) and immunofluorescence with the NuMA SPN-3 antibody (D, F) of control HeLa cells (C, D) and after $\mathrm{HU}+$ St treatment (E, F). Note the overlap between the Hoechst and the NuMA stain in control cells and the complementarity in treated cells. $(G, H)$ Hoechst $(G)$ and TUNEL $(H)$ labeling of the same HU + St-treated cells. Note the colocation of the Hoechst and TUNEL stain in the top two cells, and the lack of TUNEL signal in the lower cell which has normal Hoechst staining. Magnification: $A$ and $B, \times 135 ; C-F, \times 1500 ; G$ and $H, \times 1000$. 
A

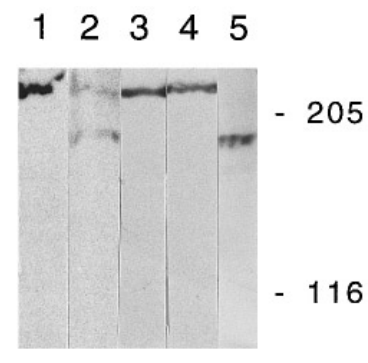

B

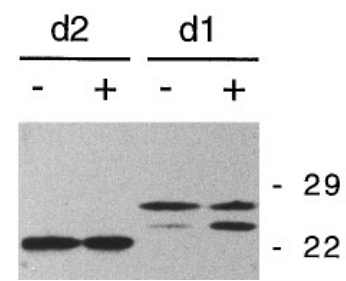

FIG. 3. Immunoblot detection of NuMA. (A) Endogenous NuMA in HeLa cells with SPN-3 antibody. Lane 1, control cells; lane 2, cells treated with $\mathrm{HU}+\mathrm{St}$; lane 3, cells treated only with $\mathrm{HU}$; lane 4, cells treated only with St; lane 5, cells treated with TNF $\alpha+$ cycloheximide. (B) Recombinant $\mathrm{d} 1$ and $\mathrm{d} 2$ NuMA detected with SPN-7 antibody in $\mathrm{BHK}$ cells without $(-)$ or with $(+) \mathrm{HU}+$ St treatment. $4 \%$ (A) and $12 \%$ (B) polyacrylamide gels. Molecular standards in kDa are indicated on the right.

detected in cells treated with either HU (lane 3) or St alone (lane 4). HeLa cells induced to enter apoptosis through treatment with TNF $\alpha$ and cycloheximide revealed only the 190-kDa form (lane 5).

The 190-kDa form could result from proteolytic cleavage of FLNUMA or from expression of a shorter N UMA isoform [16]. We distinguished between these two possibilities by expressing human recombinant FLNuMA CDNA in BHK cells since only recombinant human NuMA but not endogenous hamster BHK NuMA is de tected by SPN-3 antibody (cf. [13]). HU + St treatment induced in $\mathrm{BHK}$ cells the same apoptosis-characteristic morphological and chromatin changes as in HeLa cells and resulted in the appearance of a 190-kDa fragment which reacted with the SPN-3 antibody (data not shown). Thus the 190-kDa form results from cleavage of $F L$ recombinant NuMA.

The epitope of the SPN-3 antibody is located in NuMA residues 255- 267 (Fig. 1 [17]). Since the epitope is retained in the 190-kDa fragment, (cf. Fig. 3A, lane 5 ), this fragment could be generated either by a single cleavage in the C-terminal tail domain or by a cleavage in the tail domain combined with a second cleavage in the $\mathrm{N}$-terminal 255 residues. Therefore, the $\mathrm{HU}+\mathrm{St}$ treatment was repeated on BHK cells transfected with the $d 1$ and $d 2$ NuMA human CDNA mutants shown in Fig. 1 . $\mathrm{d} 1$ is truncated at residue 1725 and $\mathrm{d} 2$ is truncated at residue 1700 in the tail domain. Both $\mathrm{d} 1$ and d2NuMA have an internal deletion of residues 7 to
1502 introduced to increase the polypeptide separation in the immunoblotting experiment. Since the SPN-3 epitope is deleted in the $\mathrm{d} 1$ and $\mathrm{d} 2$ constructs, the blot was stained with SPN-7 antibody which recognizes an epitope between NuMA residues 1600 and 1700 (Fig. 1 [17]). Figure 3B shows clearly that d1NuMA was cleaved upon apoptosis, while d2NuMA retained its initial molecular weight. Thus the apoptotic cleavage site is located in the NuMA tail domain between residues 1701 and 1725, i.e., in the sequence ${ }_{1701}$ QVATDALKSREPQAKPQLDLSIDSL ${ }_{1725}$ [18]. Because the DA in this sequence is a potential ICE protease site [19], a point mutation in FLNuMA was constructed. However, changing $D$ to $G$ at position 1705 did not prevent cleavage under the conditions used in Fig. 3A.

\section{Inhibition of Apoptosis and NuMA Cleavage through Proteolytic I nhibitors}

Proteolytic inhibitors such as TPCK, TLCK, E-64, and ICE inhibitor II were tested for effects on apoptosis induction and on NuMA cleavage in HeLa cells. Only TPCK affected the kinetics of morphological changes. Cell retraction preceding cell rounding, usually detected 30 min after staurosporine addition, was delayed until $5 \mathrm{~h}$ in the presence of TPCK. TPCK was also the only protease inhibitor which prevented proteolytic cleavage of N uMA after $8 \mathrm{~h}$ of staurosporine treatment.

\section{DISCUSSION}

Our data suggest that proteolytic cleavage of NuMA, a nuclear matrix component of interphase cells, may be important for the apoptotic process. In normal interphase cells, NuMA and DNA, as judged by Hoechst 33242 staining, are found in an overlapping distribution, whereas in apoptotic cells they have a strikingly complementary localization (Figs. 2B - 2E). Our observation of an 190-kDa apoptotic NuMA fragment (Fig. 3) is consistent with data of Weaver et al. [11] who observed a 200-kDa NuMA fragment after induction of apoptosis in thymocytes by dexamethasone, and of $\mathrm{Hsu}$ and Yeh [12] who detected a 180-kDa NuMA fragment which lacks the four kinase sites situated past residue 2015 in the C-terminal tail domain. Appearance of an 190-kDa form of NuMA is thus a general effect of apoptosis independent of the inducer stimulus, rather than a specific effect of the protein kinase inhibitor staurosporine.

U se of the recombinant NuMA derivatives $\mathrm{d} 1$ and $\mathrm{d} 2$ in transfection experiments allowed us to position the C-terminal end of the 190-kDa NuMA form between residues 1701 and 1725 of the NuMA sequence. The calculated molecular weight of a NuMA fragment spanning for example residues $1-1710$ is $188 \mathrm{kDa}$, and this compares well with the observed molecular weight of $\sim 190 \mathrm{kDa}$ in gel electrophoresis. We assume therefore 
that the stable fragment may retain the full amino terminal end. The specific proteolytic cleavage of NuMA results in the release of the C-terminal tail domain for which multiple functions have been documented [10, 13]. This region also contains the nuclear location signal [13].

A variety of proteolytic enzymes with different substrate specificities are thought to be involved in apoptosis [4, 20]. Thus far our results provide no evidence that NuMA is a substrate for the interleukin-1$\beta$-converting enzyme (ICE) which may be involved in initiating the active phase of apoptosis [4]. Thus changing amino acid residues DA into GA at positions 17051706 did not inhibit NuMA cleavage nor did the specific ICE inhibitor II prevent cleavage. Of the various protease inhibitors tested only the chymotrypsin inhibitor TPCK was effective. Interestingly TPCK delayed both the morphological changes and NuMA cleavage.

Induction of apoptosis results in the cleavage of NuMA and in the dissociation of the 190-kDa fragment from chromatin probably because of the loss of the tail sequence. Two models of nuclear structure in which NuMA plays a key role can be envisaged. First, NuMA binds to defined DNA sequences called matrix attachment regions (MARs) in vitro [21]. MARs are viewed as thelikely starting points of DNA fragmentation [22], and thus proteolytic cleavage of NuMA might expose DNA to fragmentation and induce chromatin reorganization. Alternatively, if NuMA is a key component of a putative nuclear matrix, cleavage of NuMA may be sufficient to cause the matrix to collapse and the DNA to rearrange.

\section{REFERENCES}

1. Ellis, H. M., and Horvitz, H. R. (1986) Cell 44, 817-829.

2. Raff, M. C. (1992) Nature 356, 397- 400.

Received March 6, 1997
3. Kerr, J . F. R., Winterford, C. M., and Harmon, B. V. (1994) in Cell Biology: A Laboratory Handbook (J. E. Celis, Eds.), pp. 319- 329, Academic Press, San Diego.

4. Earnshaw, W. C. (1995) Curr. Opin. Cell Biol. 7, 337-343.

5. Zweyer, M., Bareggi, R., Grill, V., Soranzo, M. R., Marugg, R. A., Riederer, B. M., Narducci, P., and Martelli, A. (1995) Exp. Cell Res. 221, 27- 40.

6. Tinnemans, M.M.F.J., Lenders, M.-H. J.H., ten Velde, G. P. M., Ramaekers, F. C. S., and Schutte, B. (1995) Eur. J . Cell Biol. 68, 35- 46.

7. Kaufmann, S. H. (1989) Cancer Res. 49, 5870-5878.

8. Voelkel-J ohnson, C., Entingh, A. J ., Wold, W. S. M., Gooding, L. R., and Laster, S. M. (1995) J . Immunol. 154, 1707- 1716.

9. Kallajoki, M., Weber, K., and Osborn, M. (1991) EMBO J . 10, $3351-3362$.

10. Cleveland, D. W. (1995) Trends Cell Biol. 5, 60-64.

11. Weaver, V. M., Carson, C.E., Walker, P. R., Chaly, N., Lach, B., Raymond, Y., Brown, D. L., and Sikorska, M. (1996) J . Cell Sci. 109, 45-56.

12. Hsu, H.-L., and Yeh, N.-H. (1996) J . Cell Sci. 109, 277- 288.

13. Gueth-Hallonet, C., Weber, K., and Osborn, M. (1996) Exp. Cell Res. 225, 207-218.

14. Chen, C., and Okayama, H. (1987) Mol. Cell. Biol. 7, 27452752.

15. Meikrantz, W., Gisselbrecht, S., Tam, S. W., and Schlegel, R. (1994) Proc. Natl. Acad. Sci. USA 91, 3754- 3758.

16. Tang, T.K., Tang, C. C., Chen, Y., and Wu, C. (1993) J . Cell Sci. 104, 249- 260.

17. Harborth, J., Weber, K., and Osborn, M. (1995) EMBO J . 14, 2447- 2460.

18. Yang, C. H., Lambie, E. J ., and Snyder, M. (1992) J . Cell Biol. 116, 1303- 1317.

19. Thornberry, A., Bull, H. G., Calaycay, J.R., Chapman, K. T., Howard, A. D., et al. (1992) Nature 356, 768- 774.

20. Takahashi, A., Alnemri, E.S., Lazebnik, Y.A., FernandesAlnemri, T., Litwack, G., et al. (1996) Proc. Natl. Acad. Sci. USA 93, 8395-8400.

21. Ludérus, M.E.E., den Blaauwen, J.L., de Smidt, O.J.B., Compton, D. A., and van Driel, R. (1994) Mol. Cell. Biol. 14, 6297-6305.

22. Gromora, I. I., Nielsen, O. F., and Razin, S. V. (1995) J . Biol . Chem. 270, 18685- 18690. 